\title{
Computed Tomography Findings in Patients with Seizure Disorder
}

\author{
Sumnima Acharya, ${ }^{\mathrm{a}, \mathrm{c}}$ Awadesh Tiwari, ${ }^{\mathrm{b}, \mathrm{c}}$ Amit Shrestha, ${ }^{\mathrm{a}, \mathrm{c}}$ Rupesh Sharma, ${ }^{\mathrm{a}, \mathrm{c}}$ \\ Raju Shakya ${ }^{\mathrm{d}}$
}

\begin{abstract}
:
Introduction: Seizure occurs in up to $10 \%$ of the population, whereas epilepsy is a chronic disease characterized by recurrent seizures that may affect up to $2 \%$ of the population. Modern neuroimaging is useful in diagnosis of abnormalities underlying the epilepsies, but the information provided by imaging techniques can also contribute to proper classification of certain epileptic disorders and can delineate the genetics of some underlying syndromes. Neuroimaging is even more important for those patients who have medically intractable seizures. This study was carried out to establish different etiologies of seizures, to correlate the clinical data and radiological findings in cases of seizure, and to identify the common etiologies in different types of seizures. Methods: This was a retrospective hospital-based study conducted in the Department of Radiodiagnosis of Lumbini Medical College Teaching Hospital. Records of patients of past two years, admitted in any department of the hospital with history of seizure disorder and underwent a Computed Tomography (CT) of brain were included. The CT patterns were assessed and the data were tabulated and statistically analyzed. Results: There were a total of 480 cases out of which $263(55 \%)$ were male and 217 (45\%) were female with M:F ratio of 1.2:1. Generalized seizure was more frequent than partial seizure in both gender. In 274 cases of generalized seizures, CT scan findings were abnormal in 151 cases and normal finding observed in 123 cases. In 206 cases of partial seizures, 125 cases were abnormal and 81 having normal CT scan findings. Age wise distribution showed highest number $(n=218)$ of cases in young group $(<20$ yr $)$ and least number $(n=45)$ in eldest group $(>60 \mathrm{yr})$. The most common cause of seizure was calcified granuloma $(n=79$, $16.5 \%$ ) followed by neurocysticercosis (NCC, $n=64,13 \%$ ). Diffuse cerebral edema, sub-arachnoid hemorrhage, and hydrocephalus was seen only in lower age group particularly among 1-20 years. Infarct and diffuse cortical atrophy were most common cause of seizure in older age group. NCC and tuberculoma are the most common cause of partial seizure whereas cerebral infarcts, hemorrhage, malignancy, diffuse cortical atrophy are the most common cause of complex seizure. Few rare diseases like Fahr disease and tuberous sclerosis were also found in CT scan of seizure patients. Conclusion: NCC and tuberculoma are the most common cause of partial seizure whereas cerebral infarcts, hemorrhage, malignancy, and diffuse cortical atrophy are the most common cause of complex seizure. CT scan plays an important role as a preliminary tool in radiological assessment of patients presenting with seizures.
\end{abstract}

Keywords: computed tomography • epilepsy • neurocysticercosis • seizure • tuberculoma

a - Lecturer

b - Associate Professor and Head

c - Department of Radiodiagnosis,

Lumbini Medical College Teaching Hospital, Palpa, Nepal

d - Senior Consultant Family Physician,

Lumbini Medical College Teaching Hospital, Palpa, Nepal

Corresponding Author:

Dr. Sumnima Acharya

e-mail: doctorsumnima@gmail.com

How to cite this article:

Acharya S, Tiwari A, Shrestha A, Sharma R, Shakya R. Computed Tomography findings in patients with seizure disorder. Journal of Lumbini Medical College. 2016;4(1):7-10. doi: 10.22502/jlmc.v4i1.86

\section{INTRODUCTION:}

A seizure is a finite event of altered cerebral function because of excessive and abnormal electrical discharges of the brain cells. Epilepsy is a chronic condition predisposing a person to recurrent seizures. The clinical manifestation consists of sudden and transitory abnormal phenomena which may include alterations of consciousness, motor, sensory, autonomic or psychic events, perceived by the patient or by an observer. ${ }^{1}$

Seizures are classified into different types. The classification is important because etiologic diagnosis, appropriate treatment, and 
accurate prognostication all depend on the correct identification of seizures and epilepsy. There are two main types of seizures: generalized and focal. Focal seizures are those arising within networks of a single cerebral hemisphere and may remain localized or subsequently become more widely distributed. Generalized seizures rapidly affect both hemispheres as well as both sides of the body even when caused by a "focal" lesion. Generalized seizures are further subdivided into tonic-clonic, absence, myoclonic, clonic, tonic, and atonic. ${ }^{2}$

Seizures may occur in up to $10 \%$ of the population, whereas epilepsy is a chronic disease characterized by recurrent seizures that may affect up to $2 \%$ of the population. Modern neuroimaging is useful in the diagnosis of the abnormalities underlying the epilepsies, but the information provided by imaging techniques can also contribute to the proper classification of certain epileptic disorders and can delineate the genetics underlying some syndromes. Neuroimaging is even more important for those patients who have medically intractable seizures. ${ }^{3}$

Computed tomography (CT) uses ionizing radiation and can generate excellent hard tissue imaging contrast with moderately good soft tissue resolution. CT has a number of advantages, and those include lower cost, scan speed, ready accessibility, and easy use, which provide a relatively reliable imaging modality for most patients. ${ }^{4}$ Although the use of CT for patients with epilepsy has been greatly diminished by magnetic resonance imaging (MRI), $\mathrm{CT}$ is still the technique of choice for the investigation of patients with seizures and epilepsy under certain conditions. In the neonate and young infant, CT is often of secondary or adjunctive importance, but it serves as a significant backup role to ultrasound. ${ }^{5,6}$ CT can accurately detect hemorrhage, infarctions, gross malformations, ventricular system pathologies, and lesions with underlying calcification. In older children and adults, CT is the technique of choice in the perioperative state because it can rapidly detect recent hemorrhage, hydrocephalus, and major structural changes. ${ }^{7}$

\section{METHODS:}

This was a retrospective hospital-based study conducted in Department of Radiodiagnosis of Lumbini Medical College Teaching Hospital, Palpa, Nepal. The secondary data collection was done throughout the month of March, 2016. Medical records of patients admitted in any department of the hospital with history of seizure disorder and underwent a CT scan of brain from first of March, 2014 to $28^{\text {th }}$ of February, 2016 were included.

CT scan was done using Siemens Somatom Emotion and regular CT protocols were followed. CT protocols included supine position with head first and axial section of head. Plain CT scan with 10 $\mathrm{mm}$ slice thickness were taken and thin sections of two to five $\mathrm{mm}$ were also done in particular aspects. Contrast enhanced CT was also carried out whenever required.

Points in history and clinical examination were noted. The CT patterns were then noted. This study didn't involve traumatic skull or brain injuries. The data was tabulated in Microsoft Excel 2013 and analyzed by SPSS-21. Descriptive statistics like frequency and percentage were calculated. Chi-square test was applied to see relation between categorical data. $P$ value $<0.05$ was considered significant.

\section{RESULTS:}

There were 480 cases in total out of which 263 (55\%) were male and 217 (45\%) were female with M:F ratio of 1.2:1. Frequency of generalized and partial seizure and their relation to gender is shown in Table 1. It shows that partial and generalized seizure were equally distributed in both gender.

Out of 274 cases of generalized seizure, CT scan findings were abnormal in $151(55 \%)$ cases. Out of 206 cases of partial seizures, 125 (61\%) cases had abnormal CT scan findings. This difference was not statistically significant, $X^{2}(N=204, d f=1)=1.49$, $p=0.22$.

Table 2 shows the CT scan findings of patients with seizure and their age-group wise distribution. Most common finding was calcified granuloma ( $n=79,16.5 \%)$ and was most common in young age $(<20$ years). The second most common finding was neurocysticercosis which was found in $64(13 \%)$ cases. Neurocysticercosis was also most common in young group $(<20$ years). Other findings in decreasing order were diffuse cortical atrophy which was common in elderly ( $>60$ years) group, tuberculosis, granulomatous lesion, infarct

Table 1: Relation between gender and type of seizure $(N=480)$

\begin{tabular}{cccc}
\hline Gender & $\begin{array}{c}\text { Partial } \\
\text { seizure }\end{array}$ & $\begin{array}{c}\text { Generalized } \\
\text { seizure }\end{array}$ & Statistics \\
\hline Male & 112 & 151 & $X^{2}=0.026$ \\
Female & 94 & 123 & $d f=1, p=.87$ \\
\hline Total & $206(43 \%)$ & $274(57 \%)$ & \\
\hline
\end{tabular}


Table 2: CT scan findings of patients with seizure according to age group (years).

\begin{tabular}{lccccc}
\hline Findings & $\mathbf{2 0}$ & $\mathbf{2 1 - 4 0}$ & $\mathbf{4 1 - 6 0}$ & $\mathbf{7 6 0}$ & $\boldsymbol{n}$ \\
\hline Normal & 111 & 64 & 23 & 6 & 204 \\
Neurocysticercosis & 32 & 15 & 13 & 4 & 64 \\
Tuberculosis & 11 & 5 & 2 & 2 & 20 \\
Granulomatous lesion & 10 & 6 & 0 & 1 & 17 \\
Calcified granuloma & 31 & 28 & 17 & 3 & 79 \\
Infarct & 1 & 2 & 2 & 8 & 13 \\
\hline Hemorrhage & 1 & 0 & 5 & 1 & 7 \\
Diffuse Cortical atrophy & 6 & 6 & 11 & 14 & 37 \\
\hline Arachnoid cyst & 4 & 3 & 1 & 0 & 8 \\
\hline Gliotic changes & 2 & 2 & 3 & 2 & 9 \\
\hline Malignancy & 0 & 0 & 2 & 4 & 6 \\
\hline Arterio-venous malformation & 0 & 1 & 1 & 0 & 2 \\
\hline Tuberous scalerosis & 0 & 2 & 0 & 0 & 2 \\
Scizencephaly & 2 & 0 & 0 & 0 & 2 \\
\hline Hydrocephalous & 2 & 0 & 0 & 0 & 2 \\
\hline Focal cortical atrophy & 0 & 1 & 0 & 0 & 1 \\
\hline Focal cerebral edema & 1 & 0 & 0 & 0 & 1 \\
\hline Diffuse cerebral edema & 2 & 0 & 0 & 0 & 2 \\
\hline SAH & 2 & 0 & 0 & 0 & 2 \\
\hline Fahr Disease & 0 & 2 & 0 & 0 & 2 \\
\hline Total & $\mathbf{2 1 8}$ & $\mathbf{1 3 7}$ & $\mathbf{8 0}$ & $\mathbf{4 5}$ & $\mathbf{4 8 0}$ \\
\hline & & & & & \\
\hline
\end{tabular}

Table 3: CT scan findings in patients with seizure according to the type of seizure.

\begin{tabular}{lccc}
\hline CT Findings & $\begin{array}{c}\text { Partial } \\
\text { seizure }\end{array}$ & $\begin{array}{c}\text { Generalized } \\
\text { seizure }\end{array}$ & $\boldsymbol{n}$ \\
\hline NCC & 60 & 4 & 64 \\
\hline Tuberculosis & 17 & 3 & 20 \\
\hline Granulomatous lesion & 16 & 1 & 17 \\
\hline Calcified granuloma & 9 & 70 & 79 \\
\hline Infarct & 3 & 10 & 13 \\
\hline Hemorrhage & 0 & 7 & 7 \\
\hline Diffuse Cortical atrophy & 7 & 30 & 37 \\
\hline Malignancy & 0 & 6 & 6 \\
\hline Tuberous scalerosis & 0 & 2 & 2 \\
\hline Hydrocephalous & 0 & 2 & 2 \\
\hline Focal cortical atrophy & 1 & 0 & 1 \\
\hline Focal cerebral edema & 1 & 0 & 1 \\
\hline Diffuse cerebral edema & 0 & 2 & 2 \\
\hline SAH & 0 & 2 & 2 \\
\hline Fahr Disease & 0 & 2 & 2 \\
\hline
\end{tabular}

among others. Scizencephaly, hydrocephalous, focal cerebral edema, diffuse cerebral edema, and sub-arachnoid hemorrhage (SAH) were exclusively present in young $(<20$ years $)$ age group.
Table 3 shows the distribution of CT scan findings according to the type of seizure. Calcified granuloma, diffuse cortical atrophy, infarct, and malignancy were the common findings in patients with generalized seizure. Neurocysticercosis, tuberculosis, and granulomatous lesions were frequent findings in patients with partial seizure.

\section{DISCUSSION:}

Hospital admissions with history of seizures are common. Almost 3-9 per 1000 population of total hospital emergencies are seizure cases. Epilepsy is an important health problem in developing countries, where its prevalence can be up to 57 per 1000 population. ${ }^{8}$

Singh A. et al. conducted five years retrospective study which involve 915 cases of seizures including both generalized and partial. Out of total 915 cases 515 were male and 400 were female. Generalized seizure observed little more in male and female. This study had high prevalence of seizures in first, second, third and fourth decades with decreasing pattern with increasing age. Prevalence in first decade was low as compared to second and third decades. Tuberculoma (9.39\%) and Neurocysticercosis $(3.60 \%)$ had highest prevalence in partial seizures followed by focal cerebral edema (6.22\%) whereas diffuse Cerebral edema (4.91\%) seen with generalized seizures. Cerebral infarct was equally seen in both types of seizures. Brain tumor presented mostly with generalized seizure $(2.07 \%)$ than in partial seizures $(0.98 \%)$.

In our study, out of total 480 cases, 263 were male and 217 were female with M:F ratio of 1.2:1. Generalized seizure was observed more as compared to partial seizure in both males and females. Seizure was more common in first, second, third and fourth decades. In NCC and tuberculoma, the commonest finding was partial seizure whereas in cerebral infarct, hemorrhage, malignancy, diffuse cortical atrophy, complex seizure was common. Both studies are comparable.

In 274 cases of generalized seizures, CT scan findings were abnormal in 151 cases. In 206 cases of partial seizures, 125 cases had abnormal CT findings. Similar studies were done by different researchers in different places of India i.e. Bangalore, Mumbai, Chennai. ${ }^{9,10,11}$ Study done at these places showed high ratio of CT scan abnormalities in partial seizure. Highest prevalence occurred in the second decade of life in Sri Lanka. ${ }^{12}$ Study done at South India by Venkateswara MN. et al. showed high prevalence 
rate of seizure in second and third decades. ${ }^{13}$

Computerized tomography (CT) of the brain has been used routinely to study patients with epilepsy. In patients with the various electro-clinical types of epilepsy i.e. primary, secondary, and partial, it gave accurate information about the frequency, topography, and severity of morphological abnormalities. In various types of organic lesion like tumor, post-traumatic, post ischemic, post infectious, etc., it markedly increased the ability to establish etiology. ${ }^{14}$ Contrast enhanced images are very valuable in making a diagnosis. Although $\mathrm{CT}$ gives ionizing radiation to the patient, but its increasing wide spread availability, patient affordability, and short scan time makes it very valuable tool in diagnostic work up of a seizure patient. Its benefit outweighs the risk. CT scan plays a very important role as a preliminary tool in radiological assessment

\section{REFERENCES:}

1. Lowenstein DH. Seizures and Epilepsy. In: Kasper DL, Braunwald E, Fauci AS, Hauser SL, Longo DL, Jameson JL. (eds). Harrison's Principles of Internal Medicine (18 ed.). New York: Mc Graw- Hill Professional Publishing; 2008. chapter 369.

2. Berg AT, Berkovic SF, Brodie MJ, Buchhalter J, Cross $\mathrm{JH}$, van Emde Boas W, et al. Revised terminology and concepts for organization of seizures and epilepsies: report of the ILAE Commission on Classification and Terminology, 2005-2009. Epilepsia. 2010;51(4):676-85. doi: 10.1111/j.1528-1167.2010.02522.x. Epub 2010 Feb 26.

3. Kuzniecky RI. Neuroimaging of epilepsy: therapeutic implications. NeuroRx. 2005;2(2):384-93.

4. Sa de Camargo EC, Koroshetz WJ. Neuroimaging of ischemia and infarction. NeuroRx. 2005;2:265-76.

5. Hankey G, Davies L, Gubbay SS. Long term survival with early childhood intracerebral tumours. J Neurol Neurosurg Psychiatry. 1989;52:778-81.

6. Wyllie E, Rothner AD, Luders H. Partial seizures in children: clinical features, medical treatment, and surgical considerations. Pediatr Clin North Am. 1989;36:343-64.

7. Gastaut H, Gastaut JL. Computerized transverse axial of patients presenting with seizure. In about half of the seizure patients, it is able to diagnose or suggest the abnormality. It is valuable in making a diagnosis particularly in developing country like Nepal, where infections like NCC and TB are most important causes of seizures.

\section{CONCLUSION:}

Computerized transverse axial tomography is one of the important investigations to rule out the etiological causes of seizures. In Nepal, there is high prevalence of NCC followed by tuberculosis, which are commonly found in cases of partial seizures. Malignancy, cerebral edemas, calcifications, cerebral atrophy, hydrocephalus, sub arachnoid hemorrhage, intracerebral hemorrhage, infarction of basal ganglion, tuberous sclerosis are other common CT scan findings in patients with seizure disorder.

tomography in epilepsy. Epilepsia. 1976;17:325-36.

8. Senanayake N, Roman GC. Epidemiology of epilepsy in developing countries. Bull World Health Organ. 1993;71(2):247-58.

9. Mani KS, Rangan G. Epilepsy in the third world-Asian aspects. In: Dam M, Gram L. (eds.). Comprehensive epileptology. New York: Raven Press; 1991.p. 781-93.

10. Joshi V, Katiyar BC, Mohan PK, Misra S, Shukla GD. Profile of epilepsy in a developing country: a study of 1000 patients based on the international classification. Epilepsia. 1977;18(4):549-54.

11. Koul R, Razdan S, Motta A. Prevalence and pattern of epilepsy (Lath/Mirgi/Laran) in rural Kashmir, India. Epilepsia. 1988;29(2):116-22.

12. Baheti R, Gupta BD, Baheti R. A study of CT and EEG findings in patients with Generalized or Partial Seizures in western Rajasthan. JIACM. 2003;4(1):25-9.

13. Venkateswara MN, Anusha B. A Study on Trends in Prescribing Pattern of Anti- Epileptic Drugs in Teritiary Care Teaching Hospital. IJCP Sciences. 2012;3(2):25-31.

14. Gastaut H, Gastaut JL. Computerized transverse axial tomography in epilepsy. Epilepsia. 1976;17(3):325-36. 